\title{
Avaliação de variedades locais de mandioca indústria no Norte de Minas Gerais, em
}

\section{ambiente irrigado}

\author{
Evaluation of local varieties of cassava industry in the North of Minas Gerais, in an irrigated \\ environment
}

Evaluación de variedades locales de la industria de la yuca en el norte de Minas Gerais, en ambiente de regadío

Ednaldo Liberato de Oliveira ORCID: https://orcid.org/0000-0002-3075-8897 Instituto Federal do Norte de Minas Gerais, Brasil E-mail: ednaldo.liberato@ifnmg.edu.br

Nelson Licínio Campos de Oliveira ORCID: https://orcid.org/0000-0002-2198-5713 Instituto Federal do Norte de Minas Gerais, Brasil E-mail: nelson.oliveira@ifnmg.edu.br

Cândido Alves da Costa

ORCID: https://orcid.org/0000-0002-1859-1422 Universidade Federal de Minas Gerais, Brasil E-mail: costa@iac.ufmg.br

Josué Antunes de Macêdo

ORCID: https://orcid.org/0000-0001-7737-7509 Instituto Federal do Norte de Minas Gerais, Brasil E-mail: josue.macedo@ifnmg.edu.br

\begin{abstract}
Resumo
A avaliação de variedades de mandioca indústria em ambiente irrigado, na região do Norte de Minas Gerais é uma atividade carente de informações técnicas. Por isso, o objetivo deste trabalho foi avaliar, em três épocas de colheitas, cinco variedades de mandiocas já adaptadas às condições climáticas do Norte de Minas, em sistema de cultivo irrigado. O experimento foi realizado entre novembro/2018 a novembro/2019, em área experimental do Instituto Federal do Norte de Minas Gerais, Campus Januária. O experimento foi delineado em blocos casualizados, com três repetições. Foram avaliados cinco genótipos locais de mandioca para indústria: castelão, IAC-12, Canela de Ema, Projetinho e Olho Roxo. As parcelas experimentais foram compostas por quatro linhas com dez plantas, em espaçamento de $1,0 \mathrm{~m}$ entre linhas e $0,50 \mathrm{~m}$ entre plantas. A irrigação foi por aspersão convencional. As colheitas ocorreram aos 8,10 e 12 meses de idade das plantas. Foram avaliadas quanto aos caracteres, produtividade de raízes, percentagem de amido nas raízes, rendimento e produtividade de farinha. Os dados foram submetidos à análise de variância individual e conjunta e ao teste de agrupamento de médias. Os resultados revelaram que a variedade Castelão, destacou-se em relação as demais em função do desempenho agronômico, uma vez que apresentou, elevadas médias de produtividade de raízes, alto teor de amido nas raízes e, como consequência, grande rendimento de farinha, quando colhido aos dez meses após o plantio.
\end{abstract}

Palavras-chave: Januária; Amido; Irrigação.

\begin{abstract}
The evaluation of cassava varieties industry in an irrigated environment in the northern region of Minas Gerais is an activity lacking technical information. Therefore, the objective of this work was to evaluate, in three harvest seasons, five varieties of cassava already adapted to climatic conditions of the North of Minas Gerais, in an irrigated cultivation system. The experiment was carried out between November/2018 and November/2019, in an experimental area of the Federal Institute of The North of Minas Gerais, Campus Januária. The experiment was designed in randomized blocks, with three repetitions. Five local cassava genotypes for industry were evaluated: castelão, IAC-12, Canela de ema, Projetinho and Olho Roxo. The experimental plots were composed of four rows with ten plants, in spacing of $1.0 \mathrm{~m}$ between rows and $0.50 \mathrm{~m}$ between plants. Irrigation was by conventional sprinkler. The harvests took place at 8,10 and 12 months of age of the plants. Characteristics, root yield, percentage of starch in the roots, yield and flour yield were evaluated. Data were submitted to individual and joint analysis of variance and to the mean grouping test. The results revealed that the Castelão variety stood out in relation to the others in terms of its
\end{abstract}


agronomic performance, since it presented high average root productivity, high starch contents in the roots and, as a consequence, high flour yield, when harvested ten months after planting.

Keywords: Januária; Starch; Irrigation.

\begin{abstract}
Resumen
La evaluación de variedades industriales de yuca en ambiente de regadío en el norte de Minas Gerais es una actividad que carece de información técnica. Por lo tanto, el objetivo de este trabajo fue evaluar, en tres temporadas de cosecha, cinco variedades de yuca ya adaptadas a las condiciones climáticas del norte de Minas Gerais, en un sistema de cultivo de regadío. El experimento se llevó a cabo entre noviembre / 2018 a noviembre / 2019, en un área experimental del Instituto Federal del Norte de Minas Gerais, Campus Januária. El experimento se diseñó en bloques al azar, con tres repeticiones. Se evaluaron cinco genotipos de yuca local para la industria: castelão, IAC-12, Canela de Ema, Projetinho y Olho Roxo. Las parcelas experimentales estuvieron compuestas por cuatro líneas con diez plantas, con un espaciamiento de $1.0 \mathrm{~m}$ entre líneas y $0.50 \mathrm{~m}$ entre plantas. El riego se realizó por aspersión convencional. Las cosechas se realizaron a los 8, 10 y 12 meses de edad de las plantas. Se evaluaron características, rendimiento de raíces, porcentaje de almidón en las raíces, rendimiento y rendimiento de harina. Los datos se sometieron a análisis de varianza individual y conjunto y a la prueba de agrupación de medias. Los resultados revelaron que la variedad Castelão se destacó en relación a las demás en términos de desempeño agronómico, ya que presentó alta productividad promedio de raíces, alto contenido de almidón en las raíces y, como consecuencia, alto rendimiento de harina, al cosechar diez meses después de la siembra.
\end{abstract}

Palabras clave: Januária; Almidón; Riego.

\title{
1. Introdução
}

O Norte de Minas Gerais é uma região grande produtora de mandioca no Estado, mas ainda apresenta baixa produtividade devido ao desconhecimento dos produtores pelas técnicas mais modernas de cultivo. Na região da Bacia do Rio Pandeiros (envolvendo os municípios de Januária, Bonito de Minas e Cônego Marinho), as tradições na agricultura sobrevivem até hoje, como o cultivo do milho, feijão, mandioca e cana-de açúcar. Pouco se discute a respeito das dificuldades e carências inerentes ao setor do agronegócio da cadeia produtiva da farinha de mandioca na região, sobretudo no tocante à disponibilidade de informações consistentes e atualizadas que venham a contribuir de maneira significativa para o desenvolvimento do setor. A tradição do cultivo exclusivo da mandioca de sequeiro é alicerçada no paradigma de que a irrigação é economicamente inviável.

Por sua adaptação às condições do Norte de Minas Gerais, baixo custo de exploração e elevado potencial produtivo, a mandioca se constitui em uma das mais promissoras culturas, na agricultura familiar, para a região. A despeito dessa elevada importância, no entanto, seu cultivo ainda é feito sem o uso de técnicas adequadas. Vários são os motivos dessa situação. Os principais são o cultivo de fundo de quintal e de subsistência, a ocupação de mão de obra pouco qualificada, a falta de organização dos produtores, a escassez de capital dos produtores e principalmente o reduzido estímulo governamental. Somase ainda a falta de pesquisa, que vise o fortalecimento da cadeia produtiva da mandioca na Bacia do Rio Pandeiros. Até o momento, muitas informações importantes são baseadas nas próprias experiências dos produtores locais ou nas experiências de um produtor vizinho.

A cultura da mandioca produz adequadamente em regiões com precipitações entre 1.000 e 1.500 milímetros de água por ano, bem distribuídos, porém, a mandioca é muito cultivada em regiões semiáridas, com 500 a 700 mm de chuva por ano ou menos, onde é importante adequar a época de plantio, para que não ocorra deficiência de água nos primeiros meses de cultivo. O suprimento adequado de água é essencial e crítico nas fases de enraizamento e tuberização, o que ocorre do primeiro ao quinto mês após o plantio, e a falta de água, nessas fases, causam prejuízos irrecuperáveis no desenvolvimento e, consequentemente, na produção de raízes da cultura (Oliveira; Macedo \& Porto, 1982; Coelho Filho, 2020).

Estudos desenvolvidos na região do Cerrado do Brasil Central vêm apontando no sentido do elevado potencial produtivo da cultura na região, tanto no que se refere a variedades de mandioca para mesa (Fialho et al., 2009; Vieira et al., 2009; Vieira et al., 2011) quanto para indústria (Otsubo et al., 2009; Sagrilo et al., 2010; Vieira et al., 2013). Dado a 
importância do cultivo da mandioca, várias pesquisas foram realizadas no Brasil. Entre elas, podemos citar Gomes et al. (2020), Medeiros et al. (2020), Silva et al. (2020) e Sousa et al. 2021.

De modo geral há necessidade de desenvolver estudos regionais e específicos para a cultura da mandioca destinada a indústria. Muitos são os fatores (solo, clima, manejo da cultura, sistema de irrigação, dentre outros) que afetam o rendimento da cultura da mandioca. Tendo em vista a carência de estudos que abordam, principalmente, o tema irrigação na cultura da mandioca, o presente trabalho teve como objetivo avaliar variedades locais de mandioca indústria no Norte de Minas gerais, em ambiente irrigado, visando informar principalmente ao produtor os principais aspectos básicos do cultivo de mandioca irrigada, como: das suas variedades já adaptadas a região, qual é a melhor para ser usada em ambiente irrigado e qual é a melhor época de colheita.

\section{Metodologia}

O experimento foi conduzido a nível de campo no período de novembro de 2018 e novembro de 2019 (safra 2018/2019), em área experimental do Instituto Federal do Norte de Minas Gerais, Campus Januária-MG, em ambiente irrigado. No período de condução do experimento, as médias das temperaturas máximas diárias foram de $30,8^{\circ} \mathrm{C}$ e $37,7^{\circ} \mathrm{C}$, as médias das temperaturas mínimas diárias foram de $14,0{ }^{\circ} \mathrm{C}$ e $23,8{ }^{\circ} \mathrm{C}$, as médias de umidade relativa do ar foram de $42,0 \%$ e $79,10 \%$ e as precipitações acumuladas foram de $727,4 \mathrm{~mm}$, a composição física e química do solo foi de, percentagem de argila $21 \%$, percentagem de silte 5\%, percentagem de areia 74\%, pH em $\mathrm{H} 2 \mathrm{O} 6,7, \mathrm{Al}^{+++} 0,00 \mathrm{cmolc} . \mathrm{dm}^{-3}, \mathrm{Ca}^{++} 2,20 \mathrm{cmolc} . \mathrm{dm}^{-3}, \mathrm{Mg}^{++} 0,2$ cmolc.dm ${ }^{-3}, \mathrm{H}+\mathrm{Al}$ 0,56 cmolc.dm ${ }^{-3}$, fósforo $94,4 \mathrm{mg} \cdot \mathrm{dm}^{-3}$, potássio $54 \mathrm{mg} \cdot \mathrm{dm}^{-3} \mathrm{e}$ percentagem de matéria orgânica $0,6 \mathrm{~g} . \mathrm{dm}^{-}$ 3 .

O experimento foi delineado em blocos casualizados, com três repetições. Foram avaliados cinco genótipos locais de mandioca para indústria: castelão, local de coleta foi a fazenda curral novo; IAC-12, coleta em Conego Marinho; canela de ema (assentamento PA Betinho), projetinho e olho roxo, local de coleta foi fazenda picos.

As parcelas experimentais foram compostas por quatro linhas com dez plantas, em espaçamento de 1,0 m entre linhas e 0,50 m entre plantas, sendo a área útil de cada parcela constituída pelas 16 plantas centrais. Os tratos culturais (adubação e calagem) foram realizados de acordo com as recomendações técnicas da cultura da mandioca para a região e o controle do mato foi realizado por meio de capina manual (CFSEMG, 1999).

Aos 8, 10 e 12 meses após o plantio dos experimentos, foram avaliados: produtividade de raízes (PR), em toneladas por hectare (t/ha), porcentagem de amido nas raízes (PA), por meio do método da balança hidrostática (AM), descrito por Grosmann \& Freitas (1950), o rendimento de amido (RA), em porcentagem, com base na produtividade de raízes e na percentagem de amido nas raízes e a produtividade de farinha (PF), em quilos por hectare. Os dados obtidos foram submetidos à análise de variância. As médias das variáveis foram agrupadas por meio do teste Scott \& Knott, a 5\% de probabilidade de erro

O método de irrigação utilizado foi à aspersão convencional, de posse dos dados da umidade do solo correspondente à capacidade de campo (CC), ponto de murcha permanente (PMP), considerando a profundidade do sistema radicular da cultura da mandioca de $40 \mathrm{~cm}$, calculou-se a capacidade de armazenamento de água no solo (ARM), que é a lâmina de água usada como suprimento para a cultura, sendo estimada através da seguinte equação: ARM $=[(\mathrm{Ucc}-\mathrm{Upmp}) / 10] * \mathrm{f} * \mathrm{Ds} * \mathrm{Z}$, em que: ARM = lâmina d'água armazenada no solo que será usada como suprimento para a cultura $(\mathrm{mm}) ;$ Ucc $=$ teor de água do solo na capacidade de campo (\% peso), sendo estimada utilizando a equação (Arruda et al., 1987): Ucc = 3,1 + $(0,629 . \mathrm{X})-$ 0,00348 $(\mathrm{X})^{2}$; onde: $\mathrm{X}$ : teor de argila mais silte, em porcentagem. Upmp $=$ teor de água do solo no ponto de murcha permanente (\% peso), pode ser calculado conforme a equação a seguir: Upmp $=(398,9 * X) /(1308,1+X)$. Ds $=$ densidade do solo $(\mathrm{g} / \mathrm{cm} 3) ; 10=$ constante necessária para conversão de unidades; $\mathrm{f}=$ fator de disponibilidade de água no solo, ou seja, o 
limite de água que pode ser retirado do solo sem prejuízo para a cultura, Para fins práticos, o valor de "f" foi estimado em função da textura do solo: textura do solo arenosa ( $\mathrm{f}=0,4)$; média $(\mathrm{f}=0,5)$; argilosa $(\mathrm{f}=0,6)$ e $\mathrm{Z}=$ profundidade efetiva do sistema radicular, em cm, o tempo de funcionamento do sistema de irrigação, foi estimado levando em consideração a lâmina bruta e a eficiência do sistema de irrigação. Após a primeira irrigação realizou-se o manejo usando o método do turno de rega fixo de 15 dias, lâmina líquida de irrigação de $26 \mathrm{~mm}$, eficiência do sistema de irrigação de 80\%, lâmina bruta 32,5 mm, a precipitação do aspersor de $5 \mathrm{~mm}$ por hora e por fim o tempo de irrigação foi de 6,5 horas, ou seja, 6 horas e 30 minutos por posição.

\section{Resultados e Discussão}

Os fatores variedade e época de colheita influenciaram, significativamente, a produtividade de raízes tuberosas. Entretanto aos oito meses após a colheita, as médias de produtividade foram estatisticamente iguais e variaram de 34,20 a 63,6 t/ha. Vale destacar que as menores médias de produtividade foram obtidas pelas variedades IAC-12 (15,60 t/ha) e projetinho (29,40 t/ha, e a maior, pela variedade olho roxo (65,80 t/ha). Estes resultados são bem superiores à média de produtividade da região que segundo o IBGE (2011) é de 13 t/ha. Entretanto, bem abaixo do potencial produtivo da cultura que pode chegar a alcançar 90 t/ha (Cock; Wholey \& Casas, 1977).

Tabela 1: Médias de produtividade de raiz tuberosa (PR), em toneladas por hectare (t/ha), avaliadas em 5 variedades de mandioca para indústria, em três épocas de colheita (aos 8, 10 e 12 meses após o plantio), em ambiente irrigado, no município de Januária-MG. 2020.

\begin{tabular}{|c|c|c|c|c|c|}
\hline \multirow{2}{*}{$\begin{array}{c}\text { Época colheita } \\
\text { em meses }\end{array}$} & Castelão & IAC-12 & Canela de Ema & Projetinho & Olho roxo \\
\cline { 2 - 6 } & \multicolumn{7}{|c|}{ Produtividade de raiz tuberosa (t/ha) } \\
\hline 08 & $43,00 \mathrm{aA}$ & $41,80 \mathrm{aA}$ & $63,60 \mathrm{aA}$ & $37,60 \mathrm{aB}$ & $34,20 \mathrm{aB}$ \\
\hline 10 & $39,00 \mathrm{bA}$ & $46,60 \mathrm{bA}$ & $61,40 \mathrm{aA}$ & $63,40 \mathrm{aA}$ & $37,00 \mathrm{bB}$ \\
\hline 12 & $32,80 \mathrm{bA}$ & $15,60 \mathrm{bA}$ & $50,80 \mathrm{aA}$ & $29,40 \mathrm{bB}$ & $65,80 \mathrm{aA}$ \\
\hline Média & 38,26 & 34,66 & 58,6 & 43,46 & 45,66 \\
\hline $\begin{array}{l}\text { Médias seguidas pela mesma letra minúsculas na linha (variedades) e maiúscula na coluna (épocas de colheita) pertencem ao } \\
\text { mesmo grupo, a 5\% de probabilidade de erro, pelo teste de agrupamento de médias de Scott e Knott. }\end{array}$
\end{tabular}

Fonte: Dados da pesquisa.

Mota (2009), ao avaliar quatro variedades de mandioca, em Janaúba, também localizada na região Norte de Minas Gerais, obtiveram colheita precoce e produtividades elevadas. O cultivo da mandioca foi adubado, recebeu irrigações periódicas durante a sua condução e a colheita ocorreu aos oito meses após o plantio, de maneira que todas as variedades avaliadas apresentaram produtividades elevadas mesmo quando colhidas de maneira precoce. Esses resultados estão em concordância com os resultados apresentados neste experimento, nos quais todas as variedades também apresentaram produtividades consideradas elevadas, com colheita precoce. Produtividades obtidas no experimento, apresentaram produtividades média de 59,77 t/ha.

As produtividades elevadas obtidas nesse experimento podem ser justificadas pela adoção de um conjunto de práticas culturais adequadas recomendadas (stand adequado, adubação e irrigação) para a cultura da mandioca e pela escolha de variedades já adaptadas às condições climáticas da região Norte de Minas Gerais. A fertilidade do solo onde o ensaio foi conduzido deve ser considerada outro fator para justificar altas produtividades, uma vez que, de acordo com a CFSEMG (1999), a fertilidade do solo da área do ensaio pode ser classificada como alta. As altas produtividades alcançadas no ensaio 
confirmam as observações feitas por Albuquerque et al. (2008), ao afirmarem que, por meio do uso de variedades adaptadas e adoção de práticas culturais adequadas, pode-se obter produtividades de até 80 t/ha, para a cultura da mandioca.

A produtividade de raízes é um dos caracteres mais importantes para a seleção de genótipos de mandioca para indústria, em função de ser um dos fatores relacionados com os potenciais produtivos finais de farinha e de fécula de determinada variedade, no local de realização dos experimentos (Fukuda, 2005; Ceballos et al., 2002).

Com relação ao teor de amido nas raízes (TA), as variedades que apresentaram médias significativamente superiores às demais foram Castelão (42,96\%) e IAC-12 (31,73\%) nas épocas de colheitas aos dez e dose meses após o plantio, respectivamente (Tabela 2), sendo que a variedade Castelão foi a única que se destacou em ambas as épocas de colheita, apresentando a maior média quanto ao teor de amido, quando colhida aos dez meses após o plantio. Vieira et al., (2013), ao avaliarem genótipos de mandioca industriais em área de Cerrado do Noroeste de Minas Gerais, em Planaltina-DF, encontraram valores variando entre 32,29 a 27,57\%, estes valores são semelhantes aos encontrados nessa pesquisa. Segundo Conceição (1979), os teores de amido presentes nas raízes tuberosas para atenderem aos requisitos mínimos da indústria é de 30\%. Para Ceballos et al., (2002), o teor de amido das raízes é um dos caracteres mais importantes para a seleção de genótipos de mandioca para indústria em função de, juntamente, com a produtividade de raízes, determinar os potenciais produtivos de farinha e de fécula de determinado genótipo.

Tabela 2: Médias do teor de amido, em porcentagem, avaliados em 5 variedades de mandioca para indústria, em três épocas de colheita (aos 8, 10 e 12 meses após o plantio), em ambiente irrigado, no município de Januária-MG. 2020.

\begin{tabular}{|c|c|c|c|c|c|}
\hline $\begin{array}{c}\text { Época colheita } \\
\text { em meses }\end{array}$ & Castelão & IAC-12 & Canela de Ema & Projetinho & Olho roxo \\
\cline { 2 - 6 } & \multicolumn{5}{|c|}{ Teor de amido (\%) } \\
\hline 08 & $31,08 \mathrm{aB}$ & $28,56 \mathrm{aA}$ & $29,85 \mathrm{aA}$ & $29,56 \mathrm{aB}$ & $26,12 \mathrm{aA}$ \\
\hline 10 & $42,96 \mathrm{aA}$ & $29,00 \mathrm{bA}$ & $20,54 \mathrm{cB}$ & $27,24 \mathrm{bB}$ & $26,75 \mathrm{bA}$ \\
\hline 12 & $34,50 \mathrm{aA}$ & $31,73 \mathrm{aA}$ & $29,05 \mathrm{bA}$ & $34,50 \mathrm{aA}$ & $27,03 \mathrm{bA}$ \\
\hline Média & 36,18 & 29,76 & 26,48 & 30,43 & 26,33 \\
\hline
\end{tabular}

Fonte: Dados da pesquisa.

Os dados de rendimento de farinha (RF), para as cinco variedades de mandioca e três épocas de colheitas estão apresentados na Tabela 3. Houve diferença significativa entre as variedades e épocas de colheita para a característica rendimento de farinha (RF), destacando-se as variedades Castelão com um rendimento de farinha de 45,09\%, aos dez meses após o plantio, IAC-12 (35,12\%) e Projetinho $(33,80 \%)$ aos doze meses após o plantio. Segundo Takahashi e Gonçalo (2005), a época de colheita é o fator que mais influencia o rendimento industrial. 
Tabela 3: Médias de rendimento de farinha, em porcentagem, em três épocas de colheita (aos 8, 10 e 12 meses após o plantio), em ambiente irrigado, no município de Januária-MG. 2020.

\begin{tabular}{|c|c|c|c|c|c|}
\hline \multirow{2}{*}{$\begin{array}{c}\text { Época colheita } \\
\text { em meses }\end{array}$} & Castelão & IAC-12 & Canela de Ema & Projetinho & Olho roxo \\
\cline { 2 - 6 } & \multicolumn{5}{|c|}{ Rendimento de farinha (\%) } \\
\hline 08 & $29,28 \mathrm{aB}$ & $26,27 \mathrm{aB}$ & $27,59 \mathrm{aA}$ & $27,21 \mathrm{aB}$ & $22,70 \mathrm{aA}$ \\
\hline 10 & $45,09 \mathrm{aA}$ & $26,46 \mathrm{bB}$ & $25,08 \mathrm{bA}$ & $24,11 \mathrm{bB}$ & $23,45 \mathrm{bA}$ \\
\hline 12 & $28,72 \mathrm{bB}$ & $35,12 \mathrm{aA}$ & $26,53 \mathrm{bA}$ & $33,80 \mathrm{aA}$ & $23,83 \mathrm{cA}$ \\
\hline Média & 34,36 & 29,28 & 26,40 & 28,37 & 23,32 \\
\hline
\end{tabular}

Médias seguidas pela mesma letra minúsculas na linha (variedades) e maiúscula na coluna (épocas de colheita) pertencem ao mesmo grupo, a 5\% de probabilidade de erro, pelo teste de agrupamento de médias de Scott e Knott.

Fonte: Dados da pesquisa.

Ao analisarmos, na Tabela 4 as médias de produtividade de farinha (PF), em quilogramas por hectare (kg/ha), observaram-se, de acordo com teste F, que as médias desse fator são estatisticamente iguais, ou seja, não houve efeito significativo de variedades e épocas de colheita para a característica produtividade de farinha. Todavia, destaca-se um rendimento de farinha variando de 1737,5 kg/ha, para a variedade Castelão aos dez meses após colheita e 548 kg/ha, para a variedade IAC-12 aos doze meses após colheita.

Tabela 4: Médias de produtividade de farinha (PF), em hectare (kg/ha), avaliados em 5 variedades de mandioca para indústria, em três épocas de colheita (aos 8, 10 e 12 meses após o plantio), em ambiente irrigado, no município de Januária-MG. 2020.

\begin{tabular}{|c|c|c|c|c|c|}
\hline \multirow{2}{*}{$\begin{array}{c}\text { Época colheita } \\
\text { em meses }\end{array}$} & Castelão & IAC-12 & Canela de Ema & Projetinho & Olho roxo \\
\cline { 2 - 6 } & \multicolumn{7}{|c|}{ Produtividade de farinha (kg/ha) } \\
\hline 08 & $1251 \mathrm{aA}$ & $1106,00 \mathrm{aA}$ & $1755,00 \mathrm{aA}$ & $1025,00 \mathrm{aA}$ & $15,76 \mathrm{aA}$ \\
\hline 10 & $1737,5 \mathrm{aA}$ & $1236 \mathrm{aA}$ & $1534,00 \mathrm{aA}$ & $1530,00 \mathrm{aA}$ & $1023,50 \mathrm{aA}$ \\
\hline 12 & $930 \mathrm{aA}$ & $548,00 \mathrm{aA}$ & $1333,00 \mathrm{aA}$ & $990,50 \mathrm{aA}$ & $1698,00 \mathrm{aA}$ \\
\hline Média & 1306,00 & 1445,00 & 1540,50 & 1181,50 & 1165,00 \\
\hline $\begin{array}{l}\text { Médias seguidas pela mesma letra minúsculas na linha (variedades) e maiúscula na coluna (épocas de colheita) pertencem ao } \\
\text { mesmo grupo, a 5\% de probabilidade de erro, pelo teste de agrupamento de médias de Scott e Knott. }\end{array}$
\end{tabular}

Fonte: Dados da pesquisa.

Com base nos resultados obtidos, é possível afirmar que, a região de Januária-MG apresenta potencial para o cultivo de mandioca indústria irrigada, uma vez que no experimento foram obtidas: média de produtividade de raízes superior a 63 t/ha, o que é muito superior à média de produtividade de raízes de mandioca na região do Cerrado, de 13 t/ha; média de teor de amido nas raízes superior a 30\%; médias de rendimento de farinha $45 \%$ o que corresponde no caso do experimento em questão a 1737,50 .quilos de farinha. A variedade Castelão, destacou-se em relação as demais em função do desempenho agronômico apresentado, uma vez que apresentou, elevadas médias de produtividade de raízes, elevados teores de amido nas raízes e, como consequência, elevado rendimento de farinha, quando colhido aos dez meses após o plantio. Destacamos que a produção de farinha é uma opção para estocar a produção e ganhar tempo para futuras negociações e comercializações.

\section{Conclusão}

De maneira geral, as variedades de mandioca avaliadas apresentam boas características agronômicas para o cultivo em Januária (MG), em ambiente irrigado. 
A variedade Castelão se destacou das demais por apresentar maior porcentagem de amido e rendimento de farinha, bem como elevada produtividade de raiz.

Na região do Norte de Minas Gerais é possível colher mandioca para a produção de farinha e amido aos dez meses após o plantio em sistema de cultivo irrigado.

\section{Agradecimentos}

Agradecemos à Fundação de Amparo à Pesquisa do Estado de Minas Gerais (FAPEMIG) pelo apoio financeiro.

\section{Referências}

Albuquerque, J. A. A., Sediyama, T., Silva, A. A., Carneiro, J. E. S., Cecon, P. R., \& Alves, J. M. A. (2008). Interferência de plantas daninhas sobre a produtividade da mandioca (Manihot esculenta). Planta Daninha, 26(2), 279-289.

Ceballos, H., \& de la Cruz Aparicio, G. A. (2002). Taxonomía y morfología de la yuca. OSPINA, Bernardo y CEBALLOS, Hernán. Org. La yuca en el tercer milênio: Sistemas modernos de producción, procesamiento, utilización y comercialización. Cali, Colômbia: Centro Internacional de Agricultura Tropical; Consorcio Latinoamericano y de Caribe de Apoyo a la Investigación y Desarrolo de la Yuca; proyecto IP_3 Mejoramiento de Yuca. 16-31.

CFSEMG - Comissão de Fertilidade de Solo do Estado de Minas Gerais (1999). Recomendações para o uso de corretivos e fertilizantes em Minas Gerais: $5^{a}$ aproximação. Lavras, CFSEMG, 1-359.

Cock, J. H., Wholey, D., \& de las Casas, O. G. (1977). Effects of spacing on cassava (Manihot esculenta). Experimental agriculture, $13(3)$, 289-299.

Coelho Filho, M. A. (2020). Irrigação da cultura da mandioca. Embrapa Mandioca e Fruticultura-Comunicado Técnico 172. Cruz das Almas, BA, EMPRAPA, 1-12

Conceição, A. J. (1979). A mandioca. Cruz das Almas, UFBA/EMBRAPA/BNB/BRASCAN NORDESTE. 1-322.

Fialho, J. F., Vieira, E. A., Silva, M. S., de Paula-Moraes, S. V., Fukuda, W. M. G., dos Santos Filho, M. O. S., \& Silva, K. N. (2009). Desempenho de variedades de mandioca de mesa no Distrito Federal. Current Agricultural Science and Technology, 15(1-4).

Fukuda, W. M. G. (2005). Embrapa pesquisa mandioca para indústrias de amido. Revista ABAM, 11 (3).

Gomes, D. A., do Amaral, L. S., de Almeida Ferreira, D. S., Moreira, G. R., Gomes-Silva, F., da Costa, M. L. L., \& Pimentel, P. G. (2020). Caracterização de genótipos de mandioca por técnicas multivariadas. Research, Society and Development, 9(7), e252974181-e252974181.

Grosmann, J., \& Freitas, A. G. D. (1950). Determinação do teor de matéria seca pelo método de peso específico em raízes de mandioca. Revista Agronômica, 14, 75-80.

IBGE - Instituto Brasileiro de Geografia e Estatística (2011). Sistema IBGE de recuperação automática - SIDRA.

Medeiros, R. M., Saboya, L. M. F., de França, M. V., de Holanda, R. M., \& de Araújo, W. R. (2020). Aptidão climática da mandioca para o Estado de Pernambuco-Brasil. Research, Society and Development, 9(11), e279119376-e279119376.

Mota, A. D. S. (2009). Avaliação das silagens da parte aérea de quatro variedades de mandioca cultivadas no norte de Minas Gerais. Dissertação (Mestrado). Programa de Pós-Graduação em Produção Vegetal no Semi-árido, Universidade Estadual de Montes Claros, Montes Claros, Minas Gerais.

Oliveira, S. L., Macêdo, M. M. C., \& Porto, M. C. M. (1982). Efeito do déficit de água na produção de raízes de mandioca. Pesquisa Agropecuária Brasileira, 17(1), 121-124.

Otsubo, A. A., Brito, O. R., Mercante, F. M., Otsubo, V. H. N., Gonçalves, M. A., \& Telles, T. S. (2009). Desempenho de cultivares elites de mandioca industrial em áreas de cerrado do Mato Grosso do Sul. Semina: Ciências Agrárias, 30(4Sup1), 1155-1162.

Sagrilo, E., Vidigal Filho, P. S., Otsubo, A. A., Silva, A. D. S., \& Rohden, V. D. S. (2010). Performance de cultivares de mandioca e incidência de mosca branca no Vale do Ivinhema, Mato Grosso do Sul. Revista Ceres, 57, 87-94.

Silva, J. C. C., Bulhões, L. E. L., Espinosa, D. J. L., Santos, J. V., de Lima, C. L. C., de Almeida Cartaxo, P. H., \& de Oliveira Santos, J. P. (2020). Dinâmica da produção de Manihot esculenta Crantz no município de Arapiraca, Alagoas. Research, Society and Development, 9(8), e156985585-e156985585.

Sousa, N. F. C., Costa, T. L., Silva, C. C. B., Sousa, F. R. C., Paulino, C. G., \& da Silva Bonfim, L. O. (2021). Qualidade das farinhas de mandioca comercializadas em feiras livres na Cidade de Codó estado do Maranhão, Brasil. Research, Society and Development, 10(2), e51810212816-e51810212816.

Takahashi, M., \& Gonçalo, S. (2005). A cultura da mandioca. Paranavaí, Olímpica, 1-116.

Vieira, E. A., da Freitas Fialho, J., Silva, M. S., Fukuda, W. M. G., \& dos Santos Filho, M. O. S. (2009). Comportamento de genótipos de mandioca de mesa no Distrito Federal. Revista Ciência Agronômica, 40 (1), 113-122.

Vieira, E. A., Fialho, J. D. F., Faleiro, F. G., Bellon, G., Fonseca, K. G. D., Carvalho, L. J. C. B., \& Denke, M. L. (2011). Characterization of sweet cassava accessions based on molecular, quantitative and qualitative data. Crop Breeding and Applied Biotechnology, 11, 232-240.

Vieira, E. A., de Freitas Fialho, J., Faleiro, F. G., Bellon, G., da Fonseca, K. G., Silva, M. S., \& Carvalho, L. J. C. B. (2013). Caracterização fenotípica e molecular de acessos de mandioca de indústria com potencial de adaptação às condições do Cerrado do Brasil Central. Semina: Ciências Agrárias, 34 (2), 567581. 